The degrees of polymerization of the polymers, determined viscometrically, varied in a rather erratic manner, but were generally around 4,000 , independent of the rate of initiation. Since this value is considerably greater than the kinetic chain-length, the polymers must be markedly heterodisperse, perhaps owing to the fact that the sensitizer in these experiments was not uniformly distributed throughout the specimens. The crystals were prepared by freezing solutions of the sensitizer in the liquid monomer, and it was shown by ultra-violet absorption measurements that the first crystals to separate contained a relatively low concentration of sensitizer.

The above experiments do not establish that propagation through the crystal lattice occurs. The actual locus of polymerization is being determined by irradiating both large single crystals and thin crystal layers coupled with microscopic observation. Preliminary results indicate that the polymerization is at least partly a phase-boundary reaction.

C. H. BAMFORD

A. D. JENKINS

J. C. WARD

Research Laboratory, Courtaulds, Ltd., Maidenhead, Berks.

1 Mesrobian, R. B., Andor, P., Ballantine, D. S., and Dienes, G. J., J. Chem. Phys., 22, 565 (1954).

- Schulz, R., and Henglein, A., Angew. Chem., 67, 232 (1955). ${ }^{3}$ Restaino, A. J., Mesrobian, R. B., Morawetz, H., Ballantine, D. S.,
Dienes, G. J., and Metz, D. J., J. Amer. Chem. Soc., 78, 2939 (1956).

- Morawetz, H., and Fadner, T. A., Makromol. Chem., 34, 162 (1959).

- Lawton, E. J., Grubb, W. T., and Balwit, J. S., J. Poly. Sci., 19, 455 (1956).

- Abraham, R. J., Melville, H. W., Ovenall, D. W., and Whiffen, D. H., Trans. Farad. Soc., 54, 1133 (1938).

" See, for example, Whiffen, D. H., Makromol. Chem., 34, 170 (1959).

\section{A Monte Carlo Equation of State for Mixtures of Hard-Sphere Molecules}

Prockess in the theory of mixtures is limited by the lack of adequate approximate solutions of the classical many-body problem. Thus at densities greater than those appropriate to the virial expansion no satisfactory estimate of the properties of mixtures is possible. However, the Monte Carlo method enables the evaluation of the many-dimensional configurational integral to be performed by a sampling procedure. The method is applicable to any form of intermolecular potential.

The preliminary investigations of the properties of mixtures were made on mixtures containing two types of hard-sphere molecules with a ratio of diameters $\sigma_{\beta} / \sigma_{\alpha}=1 \cdot 667$. Systems of 32 and 64 molecules were studied, the molecules being confined in a cubic space by means of periodic boundary conditions ${ }^{1-3}$. Initially the two species of molecules were distributed randomly on a face-centred or simple cubic lattice. New configurations were generated by moving one particle at a time, and the results analysed in terms of three cumulative distribution functions, corresponding to the three types of molecular interaction in the mixture. A eheck of the method, using 'mixtures' of molecules of equal size, showed internal consistency among the distribution functions and good agreement with the results for single-component systems obtained by Wood and Jacobson ${ }^{4}$ and Alder and Wainwright ${ }^{5}$. In the case of mixtures the validity of the method was examined by comparing the results obtained from systems with different numbers of molecules and with differing distributions of the

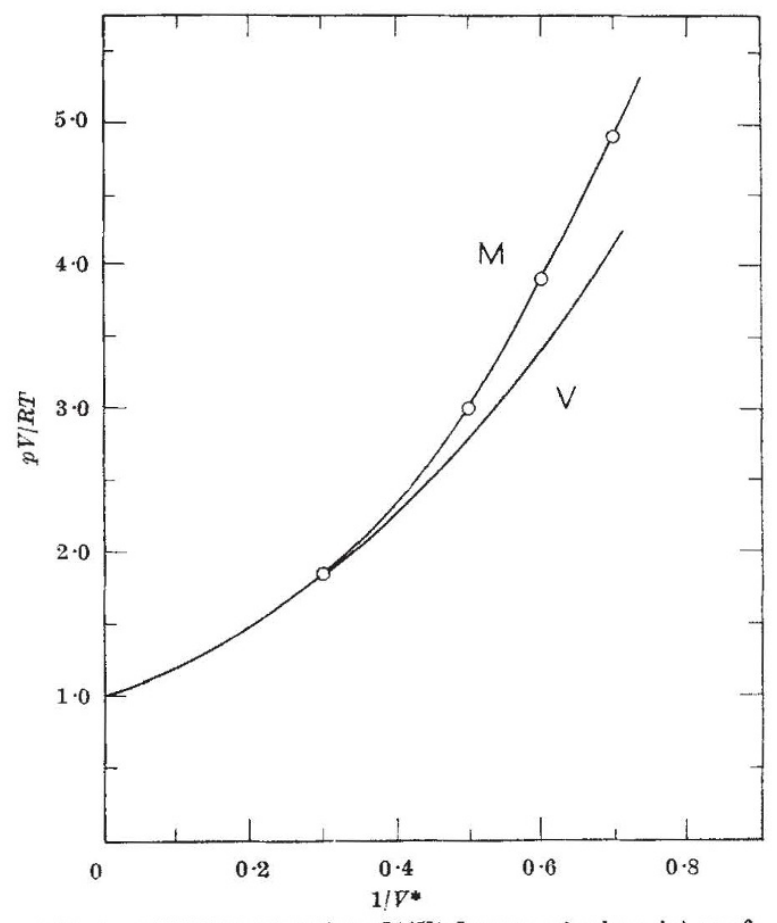

Fig. 1. $p V / R T$ as a function of $1 / V^{*}$ for an equimolar mixture of hard spheres for which $\sigma_{\beta} / \sigma_{\alpha}=1 \cdot 667$. The lower line $(V)$ represents the results of the virial expansion and the upper line $(M)$ is that obtained from the Monte Carlo calculations

two species on the initial lattice. The agreement is illustrated by the following values of $p V \mid R T$ for an equimolar mixture at a reduced density, $1 / V^{*}$, of $0 \cdot 5$ : 32 molecules, $2 \cdot 95 ; 32$ molecules, initial distributions interchanged, $3 \cdot 07 ; 64$ molecules, $3 \cdot 01$;

$$
\frac{1}{V^{*}} \text { is defined as } \frac{N_{\alpha} \sigma_{\alpha}{ }^{3}+N_{\beta} \sigma_{\beta}{ }^{3}}{V}
$$

where $N_{\alpha}$ is the number of molecules of type $\alpha$ and $\sigma_{\alpha}$ is their diameter. $V$ is the volume of the system.

Fig. 1 shows the equation of state for molecules of the given size ratio in an equimolar mixture over a range of density. The lower curve represents the results obtained from the virial expansion ${ }^{6}$, which are consistent with the Monte Carlo results at lower densities but deviate markedly at higher densities.

A full investigation is now being undertaken to determine the effect of different size ratios and to examine the thermodynamic properties of mixtures of varying composition.

The calculations were performed on the Ferranti Mercury computer at the Computing Laboratory, University of Oxford. Considerable computing time has been required, and we wish to thank Miss S. A. Tilley for her interest and assistance.

Physical Chemistry Laboratory,

$$
\text { E. B. Sмrтн }
$$

K. R. LEA

Clarendon Laboratory,

University of Oxford. April 12.

${ }^{2}$ Metropolis, Rosenbluth, Rosenbluth, Teller and Teller, J. Chem. Phys., 21, 1087 (1953).

${ }^{2}$ Rosenbluth, M. N., and Rosenbluth, A. W., J. Chem. Phys., 22, 881 (1954).

${ }^{3}$ Wood, W. W., and Parker, F. R., J. Chem. Phys., 27, 720 (1957)

4 Wood, W. W., and Jacobson, J. D., J. Chem. Phys., 27, 1207 (1957).

s Alder, B. Y., and Wainwright, T. E., J. Chem. Phys., 27, 1208 (1957).

'MeLellan, A. G., and Alder, B. J., J. Chem. Phys., 24, 115 (1956). 